\title{
Visit efsumb.org for more details on our Euroson Schools
}

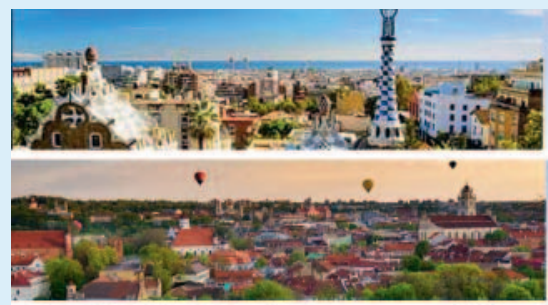

SONOANATOMY 13: Sonoanatomy of the Forearm

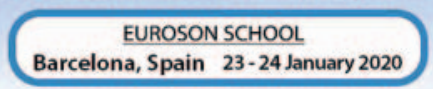

School of Multiparametric Liver Ultrasound Imaging

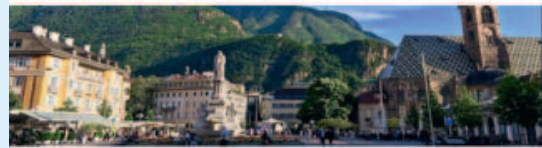

EUROSON SCHOOL
Vilnius, Lithuania $7-8$ February 2020

Musculoskeletal Sonography Course in Rheumatology

EUROSON SCHOOL
Bolzano, Italy 19-21 March 2020

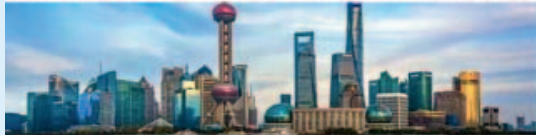

\section{CEUS Euroson School}

EUROSON SCHOOL

Shanghai, China 20 March 2020

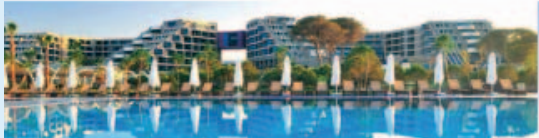

Basic Ultrasound Course

EUROSON SCHOOL

Antalya, Turkey 16-18 April 2020

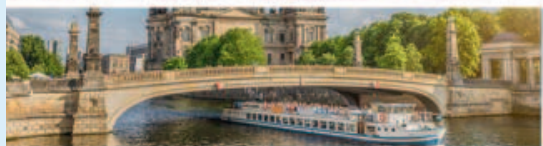

6th Euroson School for Interventional Ultrasound

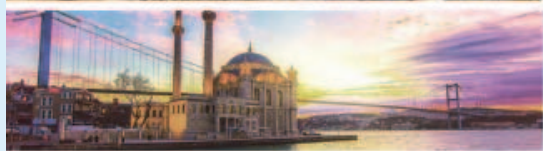

EUROSON SCHOOL

Berlin, Germany $\quad 23-25$ April 2020

Elastography, Current US Techniques and Interventional US
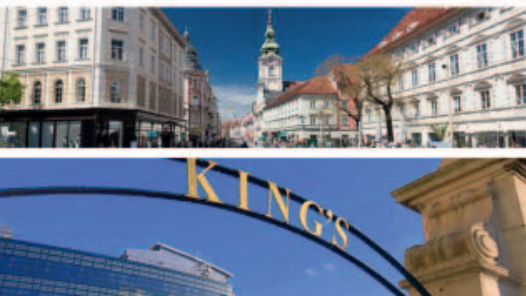

\section{EUROSON POCUS SCHOOL \\ Istanbul, Turkey 2-3 May 2020}

Musculoskeletal Sonography Course in Rheumatology

EUROSON SCHOOL
Graz, Austria $\quad 6-8$ May 2020

\section{London CEUS Ultrasound Course}

EUROSON SCHOOL
London, UK $\quad 22$ - 23 June 2020

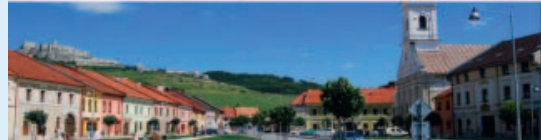

Chest Ultrasound Course (Advanced Course) EUROSON SCHOOL

Košice, Slovakia 24-27 September 2020

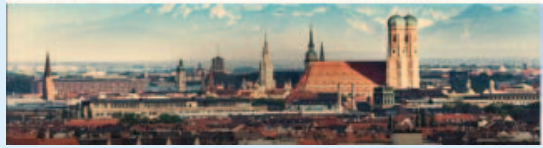

International Course in Contrast Enhanced Ultrasound Hepatic and Extrahepatic Indications EUROSON SCHOOL

Munich, Germany $14-17$ October 2020 